\title{
MARCAS FARMACÉUTICAS: UN REMEDIO PARA SU REGULACIÓN
}

\author{
FREDY SÁNCHEZ MERINO*
}

\section{INTRODUCCIÓN}

El objetivo del registro de las marcas (como registro constitutivo al fin) ha sido siempre la adquisición y reconocimiento al titular de la marca de un cúmulo de derechos que le van a permitir no solo la utilización exclusiva del signo, sino también la posibilidad de defender estos derechos frente a terceros. En consecuencia, el registro de una marca debe impedir que otro signo similar o idéntico acceda a esta misma protección, lo cual no solo obedece al respeto de los derechos exclusivos del titular previo, sino a la salvaguarda de los consumidores en el mercado, para que estos no incurran en un riesgo de confusión/asociación. Es evidente entonces que cada registro de marcas crea un micromonopolio sobre el signo en cuestión, motivo por el cual se ha de ser muy cuidadoso con respecto a qué signos acceden o no al registro, y este es precisamente uno de los roles de las oficinas de propiedad industrial en cada ordenamiento jurídico.

Para su acceso al registro, el signo debe contar con una serie de requisitos que son relativamente comunes en todas las legislaciones, y además debe estar exento de incurrir en otras tantas prohibiciones de carácter absoluto o relativo. Dentro de estas prohibiciones existe una muy particular, que en la Decisión CAN 486/2000 aparece tratada en el artículo $135 \mathrm{f}$ ) que hace referencia a la negativa de acceso al registro a aquellos signos que "consistan exclusivamente en un signo o indicación que sea el nombre genérico o técnico del producto o servicio de que se trate”.

\footnotetext{
* Abogado, magíster en Propiedad Intelectual, graduado en la Universidad de La Habana, docente de la Universidad Simón Bolívar de Barranquilla, Colombia, y consultor en Propiedad Intelectual de la Cámara de Comercio de Barranquilla. Contacto: fredex86@ gmail.com

Fecha de recepción: 4 de abril de 2015. Fecha de aceptación: 30 de abril de 2015. Para citar el artículo: Sánchez Merino, F. Marcas farmacéuticas: un remedio para su regulación. Revista La Propiedad Inmaterial n. ${ }^{\circ}$ 19, Universidad Externado de Colombia, enero-junio, 2015, pp. 53-76. DOI: 10.18601/16571959.n19.03
} 
El fundamento de esta prohibición es bastante simple: el signo genérico, como explica el propio artículo en cita, designa al producto o servicio que pretende proteger, y en consecuencia el signo per se responde a la pregunta: ¿qué es? De este modo, la denominación "bicicleta" será genérica para bicicletas, "gafa" para gafas, y así sucesivamente; de manera que sería absolutamente incomprensible que se le otorgara a un determinado sujeto el monopolio sobre una denominación que debería ser de libre disposición.

El criterio determinante para asignar a un signo el carácter de genérico es el significado que el mismo posea para los productores y consumidores según el lenguaje común y las costumbres constantes del comercio. Es importante señalar que dentro de las denominaciones genéricas se incluyen aquellas que con una grafía especial son el equivalente gráfico o fonético de una denominación genérica; y de igual forma se aprecia en el caso de los signos figurativos.

\section{A. Marcas farmacéuticas}

En este orden, resulta que las sustancias farmacéuticas pueden ser identificadas de tres maneras: con el nombre químico, el nombre genérico y la marca. Los nombres genéricos viabilizan su uso ya que las hacen más fáciles de recordar y de manipular a nivel de lenguaje por parte de un público que en su mayoría no tiene por qué dominar la terminología química o farmacéutica. La marca, como sabemos, es aquel signo (o la combinación de estos) destinado a distinguir productos y servicios de sus similares en el mercado ${ }^{1}$. A resultas de que un mismo producto pueda ser llamado de tantas maneras, no son pocos los casos en los que un nombre y otro coinciden, específicamente la marca y la denominación genérica del medicamento. Ello ocurre porque los productores prefieren usar nombres o partes de estos que cuentan con una aceptación por parte del público al que va dirigido el producto. Cuando esto ocurre se desencadenan una serie de problemas que van desde la interrupción de toda una dinámica de nombramiento de productos farmacéuticos (que explicaremos más adelante) hasta una confusión en los consumidores que entraña un riesgo bastante alto.

Por lo general, cuando una marca es idéntica a la denominación genérica del producto que está cubriendo (incluidos los farmacéuticos) es rechazada en el registro sobre la base de la prohibición absoluta antes mencionada. No obstante, pudiera darse el caso de que la marca no fuera el nombre genérico de la sustancia, y estuviera exenta de esta prohibición, pero en cambio contuviera elementos en su composición que la hicieran similar a alguna de estas denominaciones genéricas, incurriendo en alguna prohibición relativa. Entonces, además del corres-

1. Fernández-Novoa, Carlos, Tratado sobre Derecho de Marcas, Marcial Pons, Madrid, 2004. 
pondiente análisis de fondo ${ }^{2}$, el examinador deberá ser muy cuidadoso al evaluar la incursión del signo en alguna prohibición relativa, puesto que la coincidencia entre dos marcas farmacéuticas similares o idénticas, o entre una marca y una denominación común internacional (DCI), generaría sin duda un riesgo de confusión/asociación en el consumidor.

Este riesgo se encuentra matizado por la naturaleza de los productos que dicha marca pretende proteger y por los daños que una confusión respecto de los mismos pudiera ocasionar. Por ejemplo, de acuerdo a la Food and Drug Administration (FDA) de Estados Unidos ${ }^{3}$, la confusión entre marcas de medicamentos constituyó la causa de casi el 10\% de todos los errores médicos reportados en el año $2005^{[4]}$, y perjudica a aproximadamente 1.3 millones de personas cada año solo en Estados Unidos ${ }^{5}$, donde se ha evidenciado que la confusión de drogas Como FLOMAX $®$ y VOLMAX $®$ o de metilfenidato con metadona ha provocado la hospitalización e incluso la muerte de los pacientes ${ }^{6}$.

Entonces, ¿para qué asignar una marca a productos farmacéuticos si ya existen nombres genéricos? ¿Responde esto a un simple fin comercial o lucrativo por parte de las compañías farmacéuticas? Indudablemente los productores de medicamentos tienen el lucro como propósito principal, y de hecho una primera aproximación pudiera llevarnos a pensar que impidiendo el uso de marcas para medicamentos quedaría erradicado el problema. Pero la verdad es que el propósito oneroso por el que se marcan los medicamentos no va en detrimento de los fines y funciones de las marcas reconocidos en la doctrina y que aplican también para las marcas farmacéuticas; de hecho, el consumidor es más propenso a consumir medicamentos marcados 7 , y una vez que encuentra una marca de medicina que llena sus expectativas, se muestra renuente a cambiarse a otra, y menos aún a versiones genéricas de la misma, ya sea por una reacción a diferentes ingredientes inactivos o a un estado de comodidad mental; no es menos cierto que dicho

2. Aunque los daños más evidentes se producen a raíz del riesgo de confusión entre una marca y una DCI, el examinador debe ser igual de riguroso con las marcas farmacéuticas que incurran en prohibiciones absolutas fuera de constituir el nombre genérico de la sustancia que resulten particularmente perjudiciales para los consumidores, específicamente aquellas que son engañosas.

3. La Administración de Drogas y Alimentos de Estados Unidos es el órgano estatal encargado de velar por la salud pública asegurando, entre otras cosas, la eficacia de las drogas humanas y animales, productos biológicos, aparatos médicos, suministros de comida, cosméticos y productos que emiten radiación. Tomado de www.fda.gov Consultado el 30 de marzo de 2015.

4. Rados, Carol, "Drug name confussion: Preventing medication errors", FDA Consumers Magazine, julio-agosto, 2005, p. 35.

5. Ibíd.

6. Ibíd.

7. INTA (Educational Brief), The importance of pharmaceutical trademarks in protecting public health, 2007. Tomado de: www.inta.org 
grado de comodidad se perdería si los medicamentos solo pudieran identificarse por su nombre genérico.

Además, con la inclusión de la publicidad Direct-to-Consumer (DTC $)^{8}$ en muchos países, los consumidores aprenden mucho más rápido acerca de la disponibilidad y los efectos de las nuevas drogas del mercado; dicho aprendizaje sería mucho menos efectivo si tales consumidores tuvieran que manejar nombres genéricos cada vez más complejos, e incluso similares, si pertenecieran a la misma familia farmacológica, en lugar de marcas "pegadizas" y fácilmente recordables.

Por otra parte, ser capaz de reconocer el medicamento en caso de una reacción adversa es mucho más fácil si el mismo está identificado con una marca; al contrario, sería extremadamente difícil saber qué droga produce efectos adversos si todas se identifican con el mismo nombre (genérico). Tengamos en cuenta que las marcas son reparadoras de responsabilidad ${ }^{9}$, puesto que permiten rastrear productos defectuosos o de baja calidad hasta su origen, lo cual genera un cuidado extra en los productores, cuidado que no existiría si se pudieran camuflar bajo el nombre genérico del medicamento.

En el ámbito de la registrabilidad, las marcas farmacéuticas pueden incurrir en dos tipos de confundibilidad. La primera ocurre entre dos marcas similares o idénticas; la segunda, entre una marca y una DCI; aclarando que en estas últimas la confundibilidad solo puede ser denominativa, pues aunque el riesgo de confusión de manera general puede configurarse en marcas de todo tipo (siempre que concurran los requisitos necesarios), en este caso concreto la representación gráfica de las moléculas que equivalen al principio activo del medicamento sería extremadamente compleja y por lo tanto carente de distintividad. De esta forma, el riesgo de confusión o cualquier otro supuesto analizado a lo largo de este trabajo será relativo a las marcas denominativas, a menos que expresamente se aclare lo contrario.

8. "Direct-to-consumer" es un mecanismo de publicidad aplicado a productos farmacéuticos que permite hacer propaganda de estos a través de radio, televisión, internet, etc. Se originó en Estados Unidos y cobró fuerza a partir de 1976 con el caso Virgnia State Board of Pharmacy v. Virginia CItizens Consumer Council, Inc., en el cual la Corte Suprema declaró que la ley que prohibía a los farmaceutas del Estado de Virginia hacer publicidad del precio de las drogas con prescripción médica era inconstitucional, y ello sobre la base de la Primera Enmienda y la libertad de expresión comercial. El Estado de Virginia alegaba que la publicidad de los productos farmacéuticos terminaría convirtiendo al farmaceuta en un simple vendedor minorista, en lugar del experto en prescripción de drogas que en realidad debería ser. El magistrado Rhenquist en su disenso temía que la publicidad de precios de medicamentos conllevarían comerciales sin naturaleza médica, lo cual con el tiempo quedó ratificado, puesto que hoy en día las grandes farmacéuticas publicitan sus productos sin la participación de los médicos.

9. J. Thomas McCarthy, McCarthy on trademarks and unfair competition, $4 .^{a}$ ed., vol. $1,2006$. 
En relación con esto, existe en el derecho marcario otra figura que coadyuva a mantener al margen del uso aquellas marcas que en principio no son genéricas pero que, pasado un tiempo de su uso en el comercio, se vuelven genéricas: se trata de la vulgarización. Y es que si la marca es aprehendida en exceso por los consumidores y en consecuencia pierde su distintividad, se convierte en el nombre genérico del producto; así, marcas de medicamentos como la "aspirina", la "morfina" y la "heroína" ${ }^{10}$ han caducado a nivel territorial ${ }^{11}$ o global por convertirse en el nombre común con el que se designa el producto. Siendo importante apuntar que en muchos casos (como los mencionados) estas denominaciones genéricas no son $\mathrm{DCI}^{12}$ por haberse convertido en tales antes de que se implementara ese sistema por la oms, lo cual no significa que en caso de vulgarización dicha identificación no pueda ser aceptada como DCI. Lo cierto es que la vulgarización, como figura del derecho de marcas, constituye un óbice independiente y autónomo que garantiza per se que cualquier marca que se haya vulgarizado deje de serlo, incluidas aquellas que identifican medicamentos.

Una vez establecida la problemática que surge del hecho de que coincidan tanto las marcas farmacéuticas entre sí como con las DCI, procedemos a hacer un análisis del tratamiento que esta figura ha recibido en el seno de la OMS y la OMPI a fin de acercarnos a la regulación institucional de esta situación, la cual muestra una tendencia a incrementarse si no se toman las medidas adecuadas.

\section{TRATAmiento BRINDADO POR LA OMS}

A partir de una resolución ${ }^{13}$ de la Asamblea Mundial de la Salud sobre las Denominaciones Comunes Internacionales para Substancias Farmacéuticas (adoptada en mayo de 1950 por la Tercera Asamblea Mundial de la Salud) ${ }^{14}$, la oms se encarga de reunirse con los comités de nomenclatura nacionales para determinar una única denominación común que sea aceptada mundialmente, basada en la sustancia activa de cada preparación farmacéutica. Ello teniendo en cuenta que aun el nombre genérico del medicamento puede variar considerablemente de un país a otro. Estas denominaciones son declaradas como "comunes" por la oms y por lo tanto pasan al dominio público.

10. La fabricación legal masiva de la misma fue descontinuada por Bayer al descubrirse que generaba altos niveles de adicción y daños al organismo.

11. Consultado en la base de datos de la uspto.

12. La oMs solo acepta como DCI sustancias específicas y bien determinadas, no mezclas de sustancias, ni derivadas de plantas o productos homeopáticos o nombres usados durante mucho tiempo, como los mencionados arriba.

13. Resolución wHA 3.11 de la OMs, entrada en vigor en 1953.

14. Extraído de sct/1/2, Primera sesión, 13 a 17 de julio de 1998, Ginebra. 
Las denominaciones comunes se utilizan en las farmacopeas, las etiquetas, la información sobre productos, la publicidad y otras formas de promoción, la reglamentación farmacéutica y la documentación científica, y como base para determinar nombres de productos, por ejemplo los nombres de medicamentos genéricos (de fuentes múltiples). La utilización de esas denominaciones es normalmente una obligación de la legislación nacional o, en el caso de la Unión Europea, la legislación comunitaria. Gracias a los actuales mecanismos de colaboración, las denominaciones de los sistemas British Approved Names (BAN), Dénominations communes françaises (DCF), Japanese Adopted Names (JAN), United States Adopted Names (USAN) y otras denominaciones nacionales son, salvo raras excepciones, idénticas a las DCI ${ }^{15}$.

Existen determinadas normas procedimentales para la determinación de cada una de estas denominaciones. A grandes rasgos el proceso transita por la presentación de las propuestas de las denominaciones a la oms, las cuales son examinadas por un cuadro especial de expertos (el Cuadro Especial de Expertos de la oms en Farmacopea Internacional y Preparaciones Farmacéuticas) del que forman parte las secretarías o representantes de los principales comités de nomenclatura nacionales, que deben estar plenamente de acuerdo respecto a la denominación propuesta para que esta sea publicada en tal calidad en la Crónica de la oms, además de que las listas actualizadas con las DCI son enviadas a los Estados miembros de la OMS y a las comisiones de farmacopea nacionales o a otros órganos determinados por los Estados miembros. Posteriormente se abre un periodo de cuatro meses en el que cualquier persona interesada puede presentar oposición, ante lo cual la oms puede revisar nuevamente la propuesta o lograr que se retire la oposición, siendo válido aclarar que las propuestas no podrán ser confirmadas en tanto existan oposiciones vigentes, todo lo cual evidencia que la oms respeta íntegramente los derechos adquiridos por los propietarios de marcas. Para el caso de que no se presentara oposición, o se retirara luego de ser presentada, el Director General de la oms anunciará la selección de la denominación en calidad de denominación común internacional recomendada. "Al remitir las denominaciones comunes internacionales recomendadas a los Estados miembros de la oms, el Director General de la oms solicita que se reconozcan esas denominaciones en calidad de denominación común para la substancia y que los Estados miembros tomen las medidas necesarias para impedir la adquisición de los derechos de propiedad correspondientes a la denominación, incluida la prohibición de utilizar la denominación como marca o nombre comercial" 16 .

En un informe presentado al Comité Permanente sobre el Derecho de Marcas, Diseños Industriales e Indicaciones Geográficas (sCT) por la oMs, denominado

15. Tomado textualmente de sct/19/4 de julio de 2008 .

16. Ibíd. 
"Programa de denominaciones comunes internacionales (DCI)", se explica que generalmente una DCi contiene una parte común o raíz (llamada también partícula) que alude a la familia farmacológica o química a la que pertenece: por ejemplo, la terminación o sufijo “-micina” hace referencia a un tipo de antibióticos, luego las DCI se valen de expresiones adicionales para diferenciar a una DCI de las demás de su familia, como en el caso de "kanamicina" o "eritromicina". La partícula es generalmente un sufijo, pero también puede ser un prefijo o una combinación de un infijo y un sufijo. Las partículas adoptadas se indican en una publicación oficial de la oms (The use of stems in the selection of International Nonproprietary Names (INN) for pharmaceutical substances) cada dos años, y regularmente se publican apéndices, después de cada consulta de DCI. La utilización de partículas comunes permite a los médicos, los farmacéuticos, los científicos y cualquiera que trate con productos farmacéuticos, reconocer las sustancias que pertenecen a un grupo con una actividad farmacológica similar. Para poder indicar que una DCI está emparentada con otras sustancias que tienen una acción farmacológica similar se crearon partículas comunes. Actualmente, se utiliza un gran número de esas partículas comunes (algunas de las cuales se exponen como referencia en el Anexo 2), y cuando es necesario se crean otras nuevas.

Para la determinación del uso de las partículas existentes o la creación de una nueva se realizan consultas a grupos de expertos externos, puesto que depende de cada caso en concreto aplicar la misma raíz o no, dado que la misma puede tener funciones distintas pero basadas en la misma acción y que esta sea descubierta con posterioridad a la creación de la partícula.

Estas denominaciones deben ser de fácil uso en las diferentes culturas e idiomas, puesto que la oms alienta su uso en todas las etiquetas junto con el nombre comercial del medicamento. Desde que se aprobó el programa de las DCI a mediados del siglo pasado, se han asignado más de 8.500 nombres y se utilizan actualmente más de 4.000 en todo el mundo ${ }^{17}$.

Para la creación de las mismas, la oms se rige por una serie de principios que se consideran fundamentales y regulan todo el protocolo de creación de las DCI; para un mayor entendimiento los mismos aparecen relacionados en el Anexo 3.

Es decir, estas DCI constituyen (una vez que son aprobadas) el nombre genérico de las preparaciones farmacéuticas que denominan, lo cual evidencia entonces el problema de pretender registrarlas como marcas, o como parte de una marca. La prohibición absoluta antes mencionada, común a casi todas las legislaciones de propiedad industrial, impide el acceso al registro de aquellos signos que son la denominación común internacional del producto farmacéutico, y ello por tres razones básicas:

17. "La importancia del nombre", octubre de 2013, disponible en: www.who.int Consultado el 14 de marzo de 2015. 
1. La importancia elemental de que las DCI estén disponibles gratuitamente ante todo para su uso en el etiquetado de productos farmacéuticos.

2. La creación de las DCI se basa en la utilización de partículas comunes que luego forman familias de nombres según la variación del resto de la composición del producto. La utilización de una de estas partículas como marca interrumpiría dicha secuencia y en consecuencia limitaría la creación de nuevas DCI con base en la partícula utilizada.

3. El uso de estas denominaciones o parte de las mismas como marcas comerciales puede conllevar una confusión entre los profesionales de la medicina o los consumidores. El asunto es que este riesgo de confusión resulta no tener las mismas incidencias que en otros casos más regulares, pues tratándose de medicamentos, una confusión al respecto pone en riesgo la salud del consumidor. Dado que incluso aquellos fármacos con el mismo principio activo varían en el resto de su composición y tienen para determinados sujetos ciertos efectos secundarios que para otros no se producen, este es uno de los motivos por los que la oferta de productos farmacéuticos en el mercado es tan amplia: porque aún cumpliendo la misma función, están destinados a sujetos con distinto perfil.

La primera medida destinada a evitar la utilización de marcas coincidentes con DCI o sus partículas fue la emisión de una resolución de la oms solicitando a sus Estados miembros la elaboración de directrices políticas sobre el uso y protección de las DCI, así como la represión de la utilización de nombres comerciales derivados de las DCI.

En este sentido la práctica indica que la oms promueve el uso de las denominaciones genéricas en detrimento de las marcas comerciales para los productos farmacéuticos, lo cual obviamente constituiría un perjuicio para los productores; y aunque algunos países en el orden interno regulan cuestiones como el tamaño de la DCI en el etiquetado llegando a ser incluso más llamativo que la marca en sí, lo cierto es que el desuso de la marca farmacéutica en aras del nombre genérico traería consecuencias no poco graves para ese sector y el comercio en general dado el volumen masivo de signos que se utilizan en ese ámbito, lo cual hace poco probable por no decir imposible que tal práctica promovida por la oms llegue a feliz realización.

La protección pretendida por la OMS no se limita al uso de las DCI y sus partículas, dicha organización propone además evitar la utilización de elementos de la nomenclatura bioquímica (como -ferona en interferona, o -leuquina en interleuquina) en las marcas con base en un posible uso futuro de las mismas como partículas de las DCI, siendo "posible" el término clave. En efecto, tales elementos pudieran ser usados como partículas en un futuro, pero también pudieran no serlo, por lo que el veto de uso de los mismos sobre la base de un uso simplemente potencial pudiera ser visto no solo como una aplicación in extremis de la política de protección pretendida por la oMs, sino como una manifestación en contra de 
los principios registrales que rigen las prácticas de la Propiedad Industrial, e incluso las dinámicas de protección de las DCI establecidas entre la OMs y la OMPI. La implementación de estas políticas de registro entre ambas instituciones depende de una fluidez que solo se evidencia si ambas actúan en el mismo sentido, de manera de prohibir y permitir lo mismo. Es por ello que la oms busca que el tratamiento dado a las marcas por las oficinas nacionales sea el mismo que reciben las denominaciones comunes en el seno de la oms, porque si alguna de ellas permitiera un registro que otrora fuera denegado por la otra, automáticamente se estaría interrumpiendo el flujo que debe existir entre ambos extremos. Como se representa a continuación.

\section{ILUSTRACIÓN I}
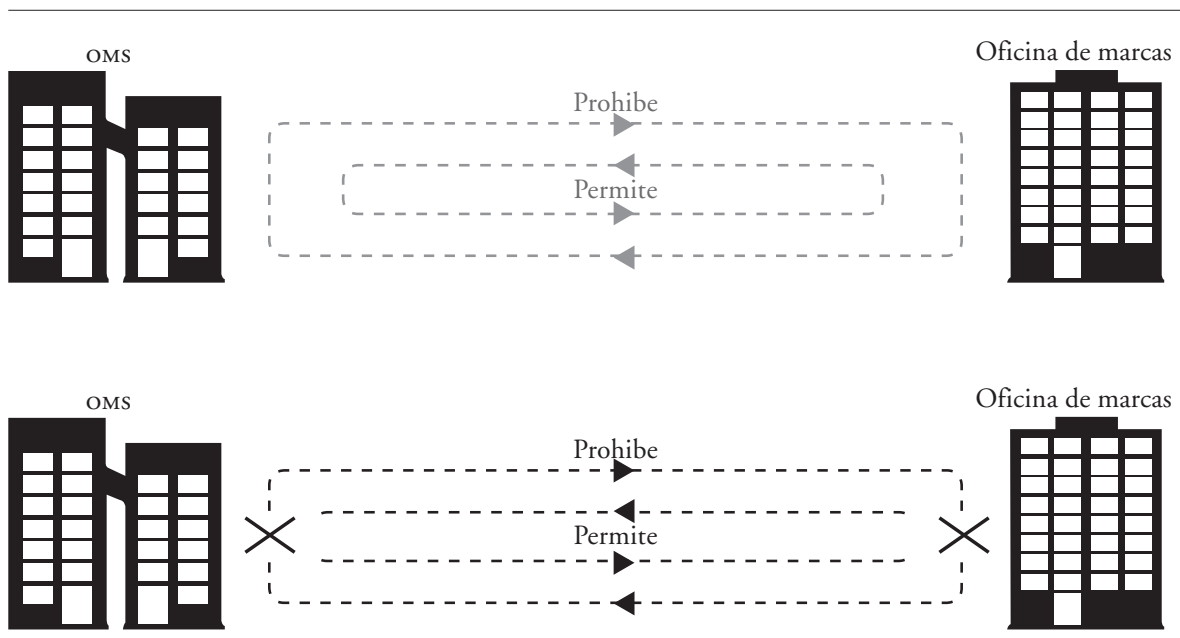

En la figura se observa cómo la denegación/permisión por ambas instituciones mantiene el flujo de denegación/registro según corresponda. En cambio, la denegación o admisión por una de las dos contradiciendo a la otra, interrumpe ipso facto la fluidez del proceso.

La dificultad radica en que la oms, como rector global de la admisión de las DCI, funciona como un filtro centralizado con poder de decisión respecto a cuáles DCI se admiten y cuáles no, pero la ompi tiene su influencia limitada sobre las Oficinas nacionales de marcas y no puede definir los mecanismos de trabajo, sino sentar bases para ello, por lo que el poder regulatorio de ambas organizaciones no es el mismo. Podemos concluir que la oms es reacia al uso de las DCi o de la raíz farmacológica que las conforma en las marcas, o de cualquier otro signo distintivo en tanto pretende que la utilización de los nombres genéricos de los medicamentos se masifique. 


\section{A. Regulación incrementada de marCas farmacéuticas}

Al margen de la búsqueda de colaboración entre las distintas instituciones encargadas de regular este tema, existe una tendencia general a incrementar la regulación de la Propiedad Intelectual en materia de marcas farmacéuticas a fin de evitar la confusión entre estas y el nombre genérico del medicamento. Esta nueva tendencia está encabezada fundamentalmente por las administraciones reguladoras de medicamentos en cada país ${ }^{18}$.

Países como Australia, Reino Unido y la Comunidad Europea, Estados Unidos, China y Canadá ${ }^{19}$ se encuentran entre los más destacados de cuantos adoptan esta nueva política.

La mayoría de las propuestas giran en torno al etiquetado y empaquetado del producto. Australia, por ejemplo, propone que los ingredientes activos del medicamento aparezcan por lo menos en 3 caras del paquete con igual tamaño y posición a la marca, y estando las primeras letras de ambas alineadas. Además plantea prohibir las marcas sombrilla, afectando entonces aquellas amparadas bajo una de estas que se vean/suenen parecido por ser de una misma familia: por ejemplo, DOLEX forte, DOLEX gripa y DOLEX niño ${ }^{20}$.

Por su parte, la Unión Europea y Reino Unido siguen una política similar a la de Australia, y aunque no es tan directa como allí respecto de las marcas sombrilla, sí establece que los nombres de productos que comparten la misma sustancia activa no pueden acceder al registro. En estos países, dada la posibilidad de un registro centralizado, la EMA (European Medicines Agency) es especialmente cuidadosa con los nombres escogidos, toda vez que van a ser válidos en varios países simultáneamente. Así mismo proponen nuevas exigencias respecto del empaquetado, sobre todo respecto de la tipografía y su espaciado, los contrastes de colores para asegurar mejor legibilidad, y el posicionamiento de la marca respecto del nombre genérico, que deben estar emparejados.

La regulación en China es bastante particular, y aunque no se asume la misma filosofía de adquisición de derechos exclusivos que en Estados Unidos, comprende el "Shang Ping Ming" 21 o nombre de propietario, que es el equivalente a una marca sin registrar. No obstante, si bien todos sus medicamentos deben llevar el

18. Norton Rose Fulbright, Increased regulation of pharmaceutical trademarks, Canadá, 2013 .

19. Ibíd.

20. Este cambio convulsionaría la tendencia marcaria de denominar formulaciones diferentes con el mismo principio activo para usos específicos, que derivaría en inversiones millonarias en re-marcado, estrategias de posicionamiento, etc. Siendo una de las causas por la cual la Therapeutic Goods Administration (TGA) en Australia y su oficina de Propiedad Industrial no se ponen de acuerdo en muchas de estas medidas.

21. Cfr. 18. 
nombre genérico, solo pueden portar nombres de propietario aquellos con nuevas estructuras químicas o nuevos ingredientes activos que aún se encuentren protegidos; o bien, compuestos medicamentosos patentados. Estos nombres deben consistir exclusivamente en caracteres chinos, y dado que solo pueden ser usados una vez por un propietario, funcionan de manera similar a las marcas, indicando el origen empresarial.

Canadá posee regulaciones (Food and Drugs Regulations) que se encargan tanto de la aprobación como del uso posterior de las marcas de productos farmacéuticos, y para ello utiliza un estricto procedimiento que comprende una guía para marcas que suenan o se ven iguales/parecidas y una revisión inicial de marcas. La guía para marcas que suenan o se ven iguales/parecidas se sustenta en tres pilares, los cuales establecen que las marcas no pueden:

1. Tener la misma o similar marca que otro producto, particularmente si dicho producto contiene diferentes ingredientes medicinales, o contiene sufijos, calificación o abreviaturas;

2. Ser similar al nombre genérico de un producto que contiene diferentes ingredientes medicinales, o

3. Nombrarlo usando una extensión de línea (según se explicó arriba con la marca sombrilla).

La revisión inicial de marcas consiste en establecer un criterio a partir de la respuesta a dos bancos de preguntas: las primeras ocho están destinadas a identificar situaciones potenciales de confusión, y una respuesta positiva a cualquiera de ellas da al traste con el registro; las preguntas siguientes se dirigen a advertir si el nombre es confuso en sí mismo, incluye abreviaturas o identifica, por ejemplo, un producto que ya no se distribuye en el país; en estos casos la solicitud de registro no se rechaza, en su lugar se le remite al solicitante para que la rectifique.

De manera general, lo explicado en este inciso ofrece una panorámica del tratamiento de este tipo de signos en algunos países con sistemas de Propiedad Intelectual muy desarrollados, y arroja dos conclusiones primarias:

1. El tratamiento de estas marcas tiende a volverse más estricto por parte de aquellas entidades encargadas de la regulación de medicamentos.

2. No existe una complementación adecuada entre las administraciones mencionadas y las oficinas de marcas, lo que genera situaciones irregulares en la protección de estos signos y de los consumidores en el mercado.

\section{TRATAMiEnTO DE LA OMPI A LAS DCI}

Los primeros acercamientos entre ambas organizaciones para la regulación de este tema se registran en el acta relativa al panorama y las cuestiones que va a tratar el (en aquel entonces denominado) Comité Permanente sobre el Derecho de Marcas, 
Dibujos o Modelos Industriales e Indicaciones Geográficas ${ }^{22}$, y aunque el tema no se incluía dentro del presupuesto propuesto, se solicitó al Comité Permanente que lo tuviera entre los temas a analizar. En ese documento se expone, en los párrafos 33 a 36, el acuerdo entre ambas organizaciones de prohibir el acceso al registro de marcas de aquellas denominaciones que coincidieran con el nombre genérico de las sustancias farmacéuticas; además se propone la realización de una encuesta (que se incluye como Anexo I), sin especificar el contenido de la misma, entre los miembros de la OMPI para determinar las prácticas de las respectivas oficinas de marcas en el análisis de aquellos signos cuya denominación era idéntica o similar al de una denominación común internacional oficial; los resultados de dicha encuesta serían debatidos posteriormente en otra reunión del sCT y se tomarían las medidas complementarias pertinentes.

La realización de dicha encuesta fue ratificada en una sesión posterior ${ }^{23}$ y se acordó la presentación de los resultados de la misma en otra sesión futura con la participación de los representantes de la oms que expresaron su preocupación por el tema aludiendo que solo en el año 1997 se conocieron más de 200 casos de posibles conflictos de DCI con registros de marcas. Recordemos que un registro de marcas, una vez concedido, puede perpetuarse si el titular lo renueva una y otra vez, dando lugar a una situación conflictiva difícil de erradicar. El cuestionario fue aplicado y en septiembre de 1999 ya se tenía respuesta de la mayoría de los países, incluido Colombia. Dicho estudio (que se encuentra en anexo al final del trabajo), a pesar de ser breve, esclareció cuestiones medulares, como cuáles países tenían en cuenta las DCI al momento de examinar una marca y basaban la denegación de registro en la coincidencia entre ambas; y cuántos recibían los listados oficiales de DCI y de qué fuente. Las respuestas a las preguntas fueron reveladoras y permitieron a la OMPI conocer que la mayoría de las oficinas encuestadas tenían en cuenta las DCI al momento del examen, así como que rechazaban el signo solicitado en caso de coincidencia; además, casi la mitad recibía los listados oficiales de DCI directamente de la oms, y casi la totalidad manifestó su disposición de comenzar a recibirlas en diversos formatos.

Aunque el cuestionario y sus resultados generales son indudablemente positivos, varias preguntas no fueron respondidas por la totalidad de los encuestados, lo cual da lugar a que los resultados y las conclusiones derivadas de los mismos sean parciales.

A raíz de los resultados obtenidos en el interrogatorio, en reuniones posteriores $^{24}$ del Comité Permanente, se convino únicamente que la ompi pediría 
asesoramiento a la oMs para poner a disposición de los miembros de la OMPI que así lo desearan, los listados en formato electrónico.

En 2006 la OMPI participó en la reunión sobre DCI para sustancias farmacéuticas del Grupo de Expertos en DCI, donde tuvieron lugar las siguientes propuestas ${ }^{25}$, aprobadas en un Comité Permanente ${ }^{26}$ posterior:

1. Poner a disposición de todas las oficinas nacionales y regionales de propiedad industrial de los Estados miembros de la OMPI la dirección del sitio web de la base de datos sobre DCI de la oms.

2. Poner a disposición de todas las oficinas un DC con las listas actualizadas de las DCI propuestas y recomendadas.

3. Que la Oficina Internacional de la OMPI informe a las oficinas nacionales y regionales de propiedad industrial de los Estados miembros de la OMPI, a través de una circular, cada vez que se publique una nueva lista de DCI propuestas y recomendadas, y que proporcione un hiperenlace con la versión electrónica de dicha lista disponible en el sitio web de la oms.

El Comité Permanente no ha abordado el tema de manera central en ninguna de sus reuniones posteriores según hemos podido constatar a través de las actas. Lo cierto es que algunas de las interrogantes ${ }^{27}$ y supuestos obstáculos obtienen un tratamiento claro por parte de la OMs pero no encuentran homologación definitiva en el órgano rector de la Propiedad Intelectual. El más acuciante de todos estos baches es si se puede usar o no la partícula de la DCI como parte de la denominación marcaria. Aunque la OMPI insta a la OMs a declararse al respecto como si no quedara claro aún, esta última ha sido meridiana al respecto, manifestando que el uso de las raíces por parte de los titulares de marcas afecta la sistematicidad que se pretende establecer con el mecanismo de creación de las DCI, puesto que el uso de dichas partículas por el propietario de un signo marcario interrumpe de lleno la posibilidad de que la familia farmacológica se continúe expandiendo. No obstante, consideramos que tal incertidumbre por parte de la OMPI y las oficinas nacionales tiene cierto fundamento debido a que no hay dudas de cómo proceder cuando la marca solicitada es idéntica a la denominación, porque estaría incursa en una prohibición absoluta (para Colombia, el art. 135 f) de la Decisión 486/2000); en cambio, cuando es similar, existe una imprecisión técnico-jurídica, puesto que las DCI son de dominio público y en consecuencia no se estarían violando derechos exclusivos previos de nadie, luego tampoco son el término genérico con que se designa el medicamento, sino un vocablo similar y en consecuencia distinto; así, la indecisión debe recaer sobre el hecho de considerar o no la evaluación de sig-

25. sст/16/9 de mayo de 2007.

26. sct/18/6 de septiembre de 2007.

27. Como el uso de partículas o la transliteración de caracteres cirílicos. 
nos con semejanza parcial, mas no en cómo proceder, pues entendemos que al respecto se aplicarían de manera normal las reglas de cotejo marcario.

En caso de que se optara por estandarizar la observación de las partículas y de la similitud en las marcas con las DCI, consideramos que además de dichas reglas de cotejo deberán tenerse en cuenta otras, dada la dinámica de adquisición que siguen los consumidores para hacerse con este tipo de productos. Teniendo en cuenta que la confusión puede darse en el paciente, el farmaceuta, el enfermero o el doctor (siendo este último el menos probable), sería prudente considerar la aplicación de pruebas de escritura para identificar la posible confusión de un medicamento con otro una vez prescrito, en aras de identificar, a través de un análisis caligráfico, si dicha confusión puede ser generada a partir de la legibilidad de la escritura. En Estados Unidos, la División de Errores Médicos de la FDA realiza una prueba de esta naturaleza en la que participan enfermeros, farmaceutas y médicos en un simulacro del proceso de prescripción de una receta, en el cual se les presenta una serie de nombres de medicamentos escritos a mano, algunos de ellos presentes en el mercado y otros no, y luego se les hace una serie de preguntas relativas al nombre de la droga, si la habían visto antes, para qué sirve, etc. En Colombia, aunque muchas instituciones prescriben por ordenador, otras tantas conservan la prescripción manual, y la denominación de los medicamentos varía en dependencia de la naturaleza de la institución que los prescriba; así, las instituciones públicas recetan medicamentos a partir de su nombre genérico y luego despachan el producto, ya sea genérico o marcado, de acuerdo con la disponibilidad, en tanto en la praxis privada es mayor la tendencia a recetar productos marcados dadas las prebendas que esta formulación continua representa para los médicos. Es válido aclarar que la eficacia de esta prueba no es muy alta, pero sería un mecanismo de control extra que coadyuvaría a la eficiencia de la evicción de la confusión entre marcas y DCI.

Respecto de aquellas DCI que se encuentren en caracteres cirílicos, solo podrán ser registradas tal cual en aquellos países donde dichos caracteres sean vistos como marcas fantasiosas, por ejemplo Colombia, puesto que la oms publica la lista acumulativa de DCI traducida en seis idiomas, entre ellos el ruso, por lo que las mismas se encuentran traducidas al cirílico de ese país, y aunque muchos alfabetos cirílicos varían entre sí, se tomará como oficial este último ${ }^{28}$.

\section{Tratamiento en Colombia}

Dado que no existen unas directrices que armonicen el examen de marcas comparadas con DCI a nivel global, cada oficina es libre de determinar si la compara-

28. sст/16/9 de mayo de 2007. Donde la delegación búlgara se manifestó de acuerdo con la traducción al cirílico ruso a pesar de que los caracteres de ambos idiomas presentan diferencias. 
ción y el correspondiente rechazo será solo para las marcas que sean idénticas a las denominaciones comunes internacionales, o también para aquellas que sean similares y que igualmente pueden suscitar un riesgo de confusión. En Colombia el registro de una marca se rechaza

... sólo si el signo cuyo registro se solicita es idéntico a una DCI. Por el contrario, el registro no se rechaza cuando el signo es semejante a una DCI; por ejemplo, cuando comienza o termina por una parte de una DCI o evoca una DCI, en la medida en que el signo no sea similar a una marca anterior hasta el punto de inducir a confusión [...] [A] unque hay un riesgo mayor de que las marcas que son similares a una DCI den lugar a confusión con otras marcas, la Oficina de Colombia admite su registro ${ }^{29}$.

Aun cuando la oms es clara respecto a que para evitar confusiones y los consiguientes riesgos para la seguridad de los pacientes, las marcas no podrán formarse a partir de las DCI y, en particular, no podrán incluir la partícula común de estas denominaciones, puesto que la utilización de una partícula común en una marca limita en gran medida la selección de otras denominaciones en una serie ${ }^{30}$.

El procedimiento actual establece que una DCI no se acepta si es similar o idéntica a una marca registrada o en trámite de registro; en cambio, en el sentido opuesto, las marcas solo se deniegan cuando son idénticas a la DCI existente, lo que genera un desequilibrio en el procedimiento, pese a que este debiera ser exactamente igual en ambos planos si de verdad se pretende crear un blindaje en esta categoría de marcas.

Un tema que genera preocupación es que la aprobación de las marcas farmacéuticas se realiza bajo la autoridad exclusiva de la Superintendencia, lo cual sería muy acertado tratándose de marcas cualesquiera; pero al ser marcas médicas su aprobación debería pasar por más de un filtro, al menos el de la sic y el de la entidad encargada de regular la circulación de productos farmacéuticos en el país, o sea el Instituto Nacional de Vigilancia de Medicamentos y Alimentos (INVIMA), cuya misión es proteger y promover la salud de la población, mediante la gestión del riesgo asociada al consumo y uso de alimentos, medicamentos, dispositivos médicos y otros productos objeto de vigilancia sanitaria. De esta forma se sobreentiende la necesidad de colaboración entre ambas entidades de manera excepcional para la aprobación de este tipo de signos y, más allá, la creación de protocolos de trabajo que den como resultado marcas farmacéuticas más seguras.

29. sct/18/10 Prov. de noviembre de 2007.

30. Programa DCI de la oms (1997). Guidelines on the Use of International Nonproprietary Names (INNs) for Pharmaceutical Substances, en wHO/PHARM s/NOM 1570 (Distribución General). 
No obstante y dada la imposibilidad de que dicha asociación entre ambas instituciones ocurra al menos de manera expedita, podrían tomarse otras medidas siguiendo el ejemplo de otros ordenamientos jurídicos que intentan darle solución a este problema. Un remedio útil sería la aplicación de un análisis reforzado por parte de la Superintendencia cuando se trate de estos productos de la clase 05; algo muy similar al "principio de precaución mayor" aplicado por la РTO ${ }^{31}$ para el análisis de las marcas farmacéuticas debido al elevado riesgo para la salud derivado de los errores de medicación suscitados por la confusión en las marcas, lo que obliga al solicitante a cumplir con estándares más elevados que demuestren que su marca no es propensa a provocar riesgo de confusión en los consumidores; si bien el nivel de dichos estándares no está especificado, toda vez que el principio en mención no está establecido por ley. Ahora bien, teniendo en cuenta que en Estados Unidos la adquisición de los derechos exclusivos de marcas se logra con el simple uso de la marca en el comercio, y por lo tanto el registro de la misma es meramente declarativo (al contrario que en Colombia), le corresponde al solicitante probar que su marca no incurrirá en riesgo de confusión; esta situación no es aplicable a nuestro ordenamiento jurídico, pero se podría adaptar si se aplicara el "principio de precaución mayor", de oficio, por la Oficina al momento del análisis de la marca, observando de manera más estricta los parámetros de evaluación del riesgo de confusión ${ }^{32}$; de esta manera se podrá argumentar que por su naturaleza los signos que distinguen determinados productos de la clase 05 de Niza se someten a un análisis reforzado por parte de la Oficina, y en consecuencia su uso en el comercio es mucho más seguro.

\section{a. Acerca del "principio de precaución mayor"}

Este principio consiste en la aplicación por las Cortes y en su caso las Oficinas de marcas, de criterios más estrictos para la determinación del riesgo de confusión, cuando dicha confusión pudiera conllevar serias consecuencias. Estas consecuencias deben consistir en un daño físico o psicológico, ya que por su naturaleza, dicha doctrina no debe ser aplicada ante el solo perjuicio económico ${ }^{33}$. El daño debe ser apreciado a partir de la no recepción del producto buscado, aun cuando el uso del producto confundido no resulte dañino en sí mismo; de tal manera que si el cliente está buscando el medicamento x y por confusión consume el producto nutricional $\mathrm{Y}$, el daño será apreciado de igual forma, porque no ha consumido

31. Oficina de Patentes y Marcas de Estados Unidos.

32. Identidad de productos o servicios; identidad de signos en cualquiera de sus variantes y percepción del consumidor.

33. Wm. \& Mary Law Review, 441, Trademarks and the concept of Greater Care, Glenwood Lab., Inc. v. American Home Products Corp., 1972. 
el producto que originalmente necesita, incluso si el producto nutricional le resultare beneficioso.

Para la aplicación de esta doctrina por lo general se consideran dos factores:

1. El grado de daño, visto según se explicó supra.

2. El tipo de producto involucrado (por lo general, medicamentos con prescripción).

Puesto que el nivel de daño apreciable es difícil de definir, cabe apuntar que el examinador debería aplicar esta doctrina a productos farmacéuticos indiscriminadamente, dado que no siendo experto en esta materia no está en condiciones de determinar de manera conclusiva si cada marca de medicamento que se pone bajo su observación tendrá efectos adversos en caso de ser confundida con otra. De esta forma, protege al consumidor de manera preventiva.

La aplicación de esta doctrina no significa que las marcas para productos farmacéuticos similares a marcas previas sean denegadas de plano, solamente que estarán sometidas a un examen de confundibilidad más riguroso.

En este orden de cosas surge un problema, y es que la oficina de marcas de la SIC no tiene autoridad para impedir el uso en el comercio de una marca que no ha sido admitida en el registro, de manera que el riesgo para los consumidores persiste aunque la marca esté desterrada del registro. Lo anterior es un motivo de peso para instar a la colaboración armónica del INVIMA y la sic en este procedimiento, tal y como se explicó supra.

Respecto del riesgo de confusión ya mencionado, la jurisprudencia europea ${ }^{34}$ propone que el mismo no se configura de igual manera que en otros casos puesto que el acceso al producto es más restringido (haciendo alusión a los medicamentos recetados), por lo que las posibilidades de que el consumidor se confunda estarían disminuidas. Planteamiento con el que estamos de acuerdo solo en parte. Tengamos en cuenta que no todos los medicamentos se obtienen por prescripción, muchos son de venta libre, y en estos casos el consumidor los adquiere de manera directa. Por otra parte, en aquellos medicamentos prescritos, el consumidor está a ciegas en la mayoría de los casos debido a su desconocimiento; entonces puede ser que la confusión no nazca en el consumidor, pero arribe a él de forma transitiva, si se tiene en cuenta que antes de llegar a sus manos ha pasado por toda una cadena de personas, en cualquiera de las cuales puede haberse suscitado dicho riesgo, siendo perfectamente loable que dicho riesgo se transmita de un sujeto a otro, afectando al usuario final.

Otro punto en el tratamiento de las DCi que no ha sido pautado es el de cómo proceder con aquellas que han sido propuestas pero no recomendadas por la oms, ya que algunos países del sistema anglosajón se basan en la determinación de si la marca es débil o no, para luego establecer si es esta o la DCI la que se pondera 
en el registro. Lógicamente estas diferencias de procedimiento solo deben ocurrir entre países con el mismo sistema de derecho. Colombia, al basarse en el principio de registro, técnicamente no admite el uso de doctrina de la marca débil en este sentido, ya que una vez que se accede al registro, el signo queda protegido, sea "débil" o no. Así las cosas, la DCI propuesta que colisione con un signo solicitado o registrado, deberá ser desestimada por la oms.

La práctica de la División de Signos Distintivos de la sic demuestra que partículas como "astina" han sido usadas en numerosas marcas para registros solicitados bajo la nueva legislación andina; otras marcas, como RELAZEPAM, caen en el mismo ejemplo; pero los ejemplos más impactantes los constituyen marcas COMO NIDAZOL y SARTAN, que se componen exclusivamente de la partícula que determina la familia farmacológica de la DCI. Todas estas marcas registradas en la clase 05 del Clasificador de Niza son evidencia de que la política de registro de la SIC transita por no valorar la utilización de las partículas de las denominaciones protegidas por la oms como causal de denegación de una solicitud de registro de marca. Lo cierto es que, más allá de las libertades de procedimiento que al respecto existen, no cabe duda de que el uso de marcas similares o idénticas a las DCI genera confusión en el consumidor, y que este riesgo es mayor dada la naturaleza de estos productos, por lo que no es idóneo el proceder de la sic en este sentido.

\section{Conclusiones}

De manera general podemos concluir que las marcas farmacéuticas presentan un tratamiento cuando menos irregular a nivel mundial, y no es distinto a nivel nacional. La OMPI no ha establecido directrices claras o impulsado la firma de Convenios o Tratados vinculantes en este sentido que sirvan para armonizar el trato que se les da a estos signos y su acceso al registro de marcas.

La manifestación de figuras como el riesgo de confusión, que para otros tipos de productos es relativamente inofensiva (por solo generar un perjuicio económico), en el caso de las marcas farmacéuticas está permeado por los riesgos potenciales para la salud de los consumidores, por lo que urge minimizar las posibilidades de que tales riesgos se configuren.

La oms manifiesta una posición bastante explícita respecto a impedir que de cualquier manera signos de este tipo sean confundidos con las DCI, pero a veces sobrepasa límites que afectarían gravemente el comercio y la industria, proponiendo abiertamente el mero uso de nombres genéricos para productos de esta naturaleza.

Por todo ello se impone la necesidad de que el registro de estos signos transite por un doble filtro donde cada entidad (Invima y la sic en el caso de Colombia) pueda hacer pleno uso de su experticia y facultades y dar lugar a un registro más adecuado, preventivo y saludable de estas marcas. 
Colombia debe asumir una posición firme respecto de los procedimientos para dicho registro, por ello nos permitimos hacer y presentar este breve trabajo a fin de sensibilizar a la academia, las administraciones y el público en general respecto de este tema de acuciante importancia.

\section{Anexo I}

Cuestionario relativo a las marcas y denominaciones comunes internacionales para sustancias farmacéuticas (DCI $)^{35}$.

1.¿Examina su Oficina solicitudes para el registro de marcas en cuanto a su incompatibilidad con denominaciones comunes internacionales y/o denominaciones comunes equivalentes adoptadas por un organismo nacional o regional?

$\begin{array}{ll}\text { Sí } & \text { No } \\ 54 & 21\end{array}$

2. Si su Oficina no lleva a cabo dicho examen, ¿pueden invalidarse o cancelarse las marcas registradas que resulten incompatibles con dichas denominaciones?

Sí No

$35 \quad 7$

3. Si su Oficina lleva a cabo dicho examen, ¿¿de qué fuente obtiene las listas sobre las que se basa el examen de las solicitudes de registro de marcas?
Organización Mundial de la Salud
Otra. Indique el nombre 36
14
Problemas en la actualización de las listas (2)
Administración Nacional de la Salud

17

4. Si su Oficina lleva a cabo dicho examen, ¿efectúa su Oficina una búsqueda manual o automática a fin de determinar posibles incompatibilidades entre las solicitudes de registro de marcas y las DCI?

$\begin{array}{ll}\text { Búsqueda manual } & \text { Búsqueda automática } \\ 40 & 14\end{array}$

35. Tomado textualmente de sct/3/7 de octubre de 1999. 
5. ¿Estaría interesado en recibir las listas que contienen las denominaciones comunes internacionales propuestas o recomendadas en formato electrónico? En caso afirmativo, ¿qué formato(s) preferiría?

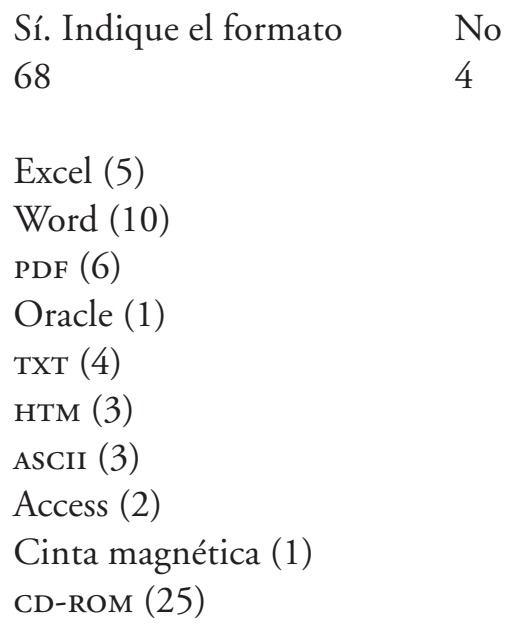

6. ¿Denegaría su Oficina el registro de una marca a causa de su incompatibilidad con una denominación común internacional? En caso afirmativo, ¿̨ajo qué condiciones?

$\begin{array}{ll}\text { Sí. Indique las condiciones } & \text { No } \\ 64^{36} & 8\end{array}$

36. Las respuestas afirmativas indicaban, generalmente, que se podía rechazar el registro de una marca en caso de conflicto con una DCI, puesto que se consideraría que dicha marca es descriptiva (cuando la marca está compuesta por una DCI propuesta o adoptada respecto de la sustancia para la cual se emplearía la marca, o contiene una DCi de esta índole) o engañosa (cuando la marca está compuesta por una DCI propuesta o adoptada respecto de una sustancia que no es aquella para la cual se emplearía la marca, o contiene una DCI de esta índole). 
ANEXO 2

CUADRO I

ALGUNAS PARTÍCULAS COMUNES QUE SE UTILIZAN EN LA SELECCIÓN DE LAS DCI ${ }^{37}$

\begin{tabular}{|c|c|}
\hline Partícula & Grupo farmacoterapéutico \\
\hline$-\mathrm{aco}$ & Antiinflamatorios del grupo del ibufenaco \\
\hline -adol/-adol- & Analgésicos \\
\hline -ast & $\begin{array}{l}\text { Antiasmáticos y antialérgicos que no actúan principalmente como antihis- } \\
\text { tamínicos }\end{array}$ \\
\hline -astina & Antihistamínicos \\
\hline -azepam & Sustancias del grupo del diazepam \\
\hline bol & Esteroides anabolizantes \\
\hline -cain- & Antiarrítmicos de Clase I, procainamida y productos del grupo de la idocaína \\
\hline -caina & Anestésicos locales \\
\hline cef- & Antibióticos derivados del ácido cefalosporánico \\
\hline -cilina & Antibióticos derivados del ácido 6-aminopenicilánico \\
\hline -conazol & Antifúngicos sistémicos del grupo del miconazol \\
\hline cort & Corticosteroides, excepto los del grupo de la prednisolona \\
\hline- coxib & Inhibidores selectivos de la ciclooxigenasa \\
\hline -entán & Antagonistas del receptor de la endotelina \\
\hline gab & Agentes gabamiméticos \\
\hline gado- & Agentes de diagnóstico del grupo del gadolinio \\
\hline -gatrán & Inhibidores de la trombina, agentes antitrombóticos \\
\hline gest & Esteroides progestágenos \\
\hline gli- & Sulfonamidas hipoglucemiantes \\
\hline io & Medios de contraste que contienen yodo \\
\hline -metacina & Antiinflamatorios del grupo de la indometacina \\
\hline -micina & Antibióticos, producidos por cepas de Streptomyces \\
\hline -nidazol & Antiprotozoarios del grupo del metronidazol \\
\hline -olol & Bloqueadores beta-adrenérgicos \\
\hline
\end{tabular}

37. Tomado de sct/19/4 de julio de 2008. 


\begin{tabular}{|l|l|}
\hline \multicolumn{1}{|c|}{ Partícula } & \multicolumn{1}{|c|}{ Grupo farmacoterapéutico } \\
\hline -oxacino & Antibacterianos del grupo del ácido nalidíxico \\
\hline -platino & Agentes antineoplásicos del grupo del platino \\
\hline -poetina & Factores sanguíneos del tipo de la eritropoyetina \\
\hline pril(at) & Inhibidores de la enzima transformadora de la angiotensina \\
\hline - profeno & Antiinflamatorios del grupo del ibuprofeno \\
\hline prost & Prostaglandinas \\
\hline -relina & Péptidos estimulantes de la liberación de hormonas hipofisarias \\
\hline -sartán & $\begin{array}{l}\text { Antagonistas del receptor de la angiotensina II, antihipertensivos (no pep- } \\
\text { tídicos) }\end{array}$ \\
\hline -vaptán & Antagonistas del receptor de la vasopresina \\
\hline vin-/-vin- & Alcaloides de la vinca \\
\hline
\end{tabular}

\section{Anexo 3}

Principios generales de orientación para formar denominaciones comunes internacionales para sustancias farmacéuticas ${ }^{38}$.

1. Las DCi deberán diferenciarse tanto fonética como ortográficamente. No deberán ser incómodamente largas, ni dar lugar a confusión con denominaciones de uso común.

2. La DCI de una sustancia que pertenezca a un grupo de sustancias farmacológicamente emparentadas deberá mostrar apropiadamente este parentesco. Deberán evitarse los nombres que puedan inducir fácilmente en el paciente sugestiones anatómicas, fisiológicas, patológicas o terapéuticas.

Estos principios fundamentales serán respetados aplicando los siguientes principios secundarios:

3. Al idear la DCi de la primera sustancia de un nuevo grupo farmacológico, deberá tenerse en cuenta la posibilidad de formar Dcr convenientes para las sustancias emparentadas que vengan a incrementar el nuevo grupo.

4. Al idear Dcr para ácidos, se preferirán las de una sola palabra; sus sales deberán denominarse sin modificar el nombre de ácido; p. ej.: "oxacilina" y "oxacilina sódica", "ibufenaco" e "ibufenaco sódico".

5. Las DCr para las sustancias que se usan en forma de sal deberán en general aplicarse a la base activa o, respectivamente, al ácido activo. Las denominaciones 
para diferentes sales o ésteres de la misma sustancia activa solamente deberán diferir en el nombre de ácido o de la base inactivos.

En los compuestos de amonio cuaternario, el catión y el anión deberán denominarse adecuadamente por separado, como componentes independientes de una sustancia cuaternaria y no como sales de una amina.

6. Deberá evitarse el empleo de una letra o un número aislados; también es indeseable el empleo de guiones.

7. Para facilitar la traducción y la pronunciación se emplearán de preferencia las letras "f" en lugar de "ph", "t" en lugar de "th", "e" en lugar de "ae" u "oe" e "i" en lugar de " $y$ "; se deberá evitar el empleo de las letras " $h$ " y " $k$ ".

8. Siempre que las denominaciones que se sugieran estén de acuerdo con estos principios, recibirán una consideración preferente las denominaciones propuestas por la persona que haya descubierto la sustancia, o la que primeramente fabrique o ponga a la venta la sustancia farmacéutica, así como las denominaciones oficialmente adoptadas en cualquier país.

9. En las dсi, la relación de grupo o parentesco (véanse los Principios Generales de Orientación, apartado 2) se indicará, en lo posible, utilizando una partícula común. En la lista siguiente se dan algunos ejemplos de estas partículas en relación con diversos grupos de sustancias, en particular los de nuevo cuño. Hay otras muchas partículas comunes en uso. Cuando la partícula no lleva ningún guion, cabe utilizarla en cualquier parte de la denominación.

\section{BiBLIOGRAFíA}

Actas del sct, sст 1/2, sст 1/6, sст 3/7, sст 3/9, sст 16/3, sст 16/9, sст 18/6, SCT $18 / 10$ prov. y SCT $19 / 4$.

FDA, Guidance related to Proprietary Names, Pharma Trade Marks Group 89 ${ }^{\text {th }}$ Conference, Chicago, 2014.

Fernández-Novoa, Carlos, Tratado sobre Derecho de Marcas, Marcial Pons, Madrid, 2004.

Fernández-Novoa, Carlos, Otero lastres, José Manuel y Botana Agra, Carlos, Manual de la Propiedad Industrial, Marcial Pons, Madrid, 2013.

Genovese, Anthony, Direct to consumer advertising in the us, Pharma Trade Marks Group 89 ${ }^{\text {th }}$ Conference, Chicago, 2014.

Grupo de Expertos en DCI, "La importancia del nombre", octubre de 2013, tomado de: www.who.int Consultado el 14 de marzo de 2015.

Hanes, Christopher, The importance of use in Commerce for Pharmaceutical trade marks in the US, Pharma Trade Marks Group 89 ${ }^{\text {th }}$ Conference, Chicago, 2014.

Herberholz, Dana, "Curing confusion, An overview of the regulatory complexities of obtaining pharmaceutical trademarks and a prescription for reform", Minn. J. L. Sci. \& Tech., 2006; 8 (1): 97-126. 
Karet, Gail, Balocco, Raffaella y Lachat, Antoinette, Non-proprietary names: Why are they precious to the industry (Panel Discussion), Pharma Trade Marks Group 89 ${ }^{\text {th }}$ Conference, Chicago, 2014.

Labrunie, JacQues, New criteria for the acceptance of pharmaceutical trademarks in Brazil, Pharma Trade Marks Group 89 ${ }^{\text {th }}$ Conference, Chicago, 2014.

McСarthy J. Thomas, Mc Carthy on trademarks and unfair competition, 4. ${ }^{\mathrm{a}}$ ed., vol.1, 2006.

Norton Rose Fulbright, Increased regulation of pharmaceutical trademarks, Canadá, 2013.

Programa DCI de la oMs, "Guidelines on the Use of International Nonproprietary Names (InNs) for Pharmaceutical Substances”, en: WHO/PHARM S/NOM 1570 (Distribución General), 1997.

Rados, Carol, "Drug name confussion: Preventing medication errors", FDA Consumers Magazine, julio-agosto, 2005, p. 35.

Resolución wHA 3.11 de la oms, entrada en vigor en 1953.

Sentencia 3529/2004 del Tribunal Supremo Español, Sala de lo Contencioso.

Swann, John P., History of the FDA, U.S. Food and Drug Administration, tomado de: www.fda.gov Consultado en marzo de 2015.

TAYlor, Kelly, Recent developments in the FDA's Review of Proprietary Names for Pharmaceuticals, Pharma Trade Marks Group 89 ${ }^{\text {th }}$ Conference, Chicago, 2014. Wm. \& Mary Law Review, 441, Trademarks and the concept of Greater Care, Glenwood Lab., Inc. v. American Home Products Corp., 1972. 